\title{
The Role of the Polymorphonuclear Leukocyte in Hyperalgesia ${ }^{1}$
}

\author{
JON D. LEVINE,, ${ }^{2,}$ JUSTIN GOODING," PAUL DONATONI,"* LUCY BORDEN, * AND EDWARD J. GOETZL‡
}

*Section of Rheumatology and Clinical Immunology, and $\ddagger$ Division of Allergy and Immunology, University of California, San Francisco, California 94143

\begin{abstract}
The results of recent studies of the mechanism of leukotriene $\mathrm{B}_{4}$-induced hyperalgesia suggest a dependence on polymorphonuclear leukocytes (PMNLs). In this study, we addressed the contribution of PMNLs to hyperalgesia evoked by the peptide chemotactic factors $\mathrm{N}$-formyl-methionyl-leucyl-phenylalanine ( $\mathrm{FMLP}$ ) and the anaphylatoxin fragment of the fifth component of the complement pathway $\left(\mathrm{C}_{5 \mathrm{a}}\right)$. Local injection of glycogen, which attracts but does not activate PMNLs, produced a marked shift to the left (toward lower concentrations) in the concentration dependence curve of fMLP-induced hyperalgesia. In addition, PMNL repletion by transfusion with syngeneic PMNLs reestablished fMLP-induced hyperalgesia in PMNL-depleted rats. Finally, supernatants from rat and human PMNLs, that had been stimulated with AMLP in vitro, produced hyperalgesia in PMNL-depleted rats. Preliminary characterization of the hyperalgesia-inducing activity released by stimulated PMNLs indicated that it is lipid in nature. The nonsteroidal anti-inflammatory indomethacin did not attenuate $C_{5 a}$ and fMLP-induced hyperalgesia. Thus, the hyperalgesia produced by $\mathrm{MLLP}$ and $\mathrm{C}_{5 \mathrm{a}}$ is similar to that produced by leukotriene $B_{4}$ in that it is dependent on PMNLs and independent of the cyclo-oxygenation of arachidonic acid. Taken together, these data suggest that structurally diverse PMNL-chemotactic factors produce hyperalgesia by a novel mechanism, involving PMNL-derived factors.
\end{abstract}

Leukotriene $\mathrm{B}_{4}\left(\mathrm{LTB}_{4}\right)$, a product of the 5-lipoxygenation of arachidonic acid, exhibits potent chemotactic and other activities for polymorphonuclear leukocytes (PMNLs) (Ford-Hutchinson et al., 1980; Goetzl and Pickett, 1980; Williams, 1983). We recently reported that intradermal injection of $\mathrm{LTB}_{4}$, in rats, also stereospecifically induces hyperalgesia, of a magnilude similar to that elicited by the same quantities of bradykinin (BK) and prostaglandin $\mathrm{E}_{2}\left(\mathrm{PGE}_{2}\right)$ (Levine et al., 1984). $\mathrm{LTB}_{4}$-induced hyperalgesia was not attenuated by prior administration of indomethacin, indicating independence from the cyclo-oxygenase pathway of arachidonic acid metabolism. Depletion of PMNLS, however, eliminated the hyperalgesia. Thus, the mechanism by which $\mathrm{LTB}_{4}$ evokes hyperalgesia is distinguished

Received January 29, 1985; Revised Aprit 1, 1985;

Accepted May 3, 1985

${ }^{1}$ This work was supported in part by Grants NS21647, AM32634, DE05369, HL31809, and Al19784 from the National Institutes of Health, and a grant from the Northern Calitornia Arthritis Foundation. J. D. L. is a fellow of the Hartford Foundation, and E. J. G. is an investigator of the Howard Hughes Medical Institute. We thank Allan Basbaum for discussions of the manuscript.

${ }^{2}$ To whom correspondence should be addressed. from that of $\mathrm{PGE}_{2}$ and BK by its dependence on PMNLs and its independence from second mediators generated by the cyclooxygenation of arachidonic acid (Levine et al., 1984).

In the present study, we report that two other potent chemoattraclants for and activators of PMNLs, namely, $\mathrm{N}$-formyl-methionyl-leucylphenylalanine (FMLP) (Showell et al., 1976) and the anaphylatoxin fragment of the fifth component of the complement pathway, $\mathrm{C}_{5 \mathrm{a}}$ (Shin et al., 1968), also produce hyperalgesia in the rat. The hyperalgesia induced by $\mathrm{FMLP}$ and $\mathrm{C}_{5 a}$ resemble that evoked by $\mathrm{LTB}_{4}$ in being independent of the cyclo-oxygenation of arachidonic acid and susceptible to attenuation by PMNL depletion. Preliminary characterization of the hyperalgesia-inducing factor produced by stimulated PMNLs suggests that it is a lipid. These data further elucidate a novel mechanism by which humoral mediators of inflammation can elicit hyperalgesia.

\section{Materials and Methods}

The experiments were performed on 250- to 350-gm male Sprague-Dawley rats (Bantin-Kingman, Fremont, CA). Hyperalgesia, or tenderness, was assessed as a decrease in the withdrawal threshold to a pressure that increases linearly with time, applied on the dorsum of a hindpaw (Randall and Selitto, 1957). Withdrawal threshold was first measured in the absence of exogenous mediators. Then, $10 \mu \mathrm{l}$ of a test substance or its vehicle werc injected intradermally into the dorsum of one hindpaw. The skin was blackened over the injection site to identify the location for further testing. Withdrawal threshold was then remeasured $10,20,40,60$, and 120 mirn post-injection. The magnitude of the hyperalgesia induced by a test substance was calculated as the percentage decrease in pressure required to elicit withdrawal, relative to the base line. In the rat, the maximum decrease in mechanical nociceptive thresholds produced by test substances using the paw withdrawal test correlates well with the analgesic potency of intradermal injections of the same substance in humans (Evans, 1964; Ferriera, 1983).

The fMLP (Sigma Chemical Co., St. Louis, MO) was initially dissolved in $10 \mu \mathrm{l}$ of dimethylsulfoxide to which, then, was added Hanks' balanced salt solution (HBSS) containing $0.1 \%$ ovalbumin and $10 \mathrm{~mm} \mathrm{HEPES} \mathrm{(pH} \mathrm{7.4).} \mathrm{The}$ final solution was sonicated for $30 \mathrm{~min}$ to ensure complete solubilization of the $\mathrm{FMLP}$. The $\mathrm{C}_{5 \mathrm{a}}$ was proparcd as proviously described and purified partially by ion-exchange chromatography and gel filtration (Goetzl and Austen, 1974); the concentration of $C_{5 a}$ in the stock solution was $0.4 \mu \mathrm{g} / \mathrm{ml}$ as assessed by specific radioimmunoassay (Upjul 11 Diagniostics, Kalamazoo, Ml). The $\mathrm{C}_{5 a}$ was dissolved in the same vehicle used to dissolve the fMLP. Indomethacin (Sigma) was dissolved in $2 \%$ sodium bicarbonate and then titrated to $\mathrm{pH} 7.2$ with phosphate buffer. Indomethacin $(2 \mathrm{mg} / \mathrm{kg})$ or an equivalent volume of vehicle was administered intraperitoneally $40 \mathrm{~min}$ prior to intradermal injection of test substances. Indomethacin produced a small increase in paw withdrawal threshold. Therefore, the threshold determined 30 min after intraperitoneal injection of indomethacin or its vehicle was used as the base line for calculating the magnitude of the hyperalgesia produced by test substances.

PMNLs in the peripheral circulation of rats were depleted by intravenous administration of hydroxyurea (E. R. Squibb and Sons, Inc., Princeton, NJ) in doses of $200 \mathrm{mg} / \mathrm{kg}$ on day 1 and $100 \mathrm{mg} / \mathrm{kg}$ on days 2 and 3, prior to hyperalgesia testing (Johnson and Malik, 1980). This protocol eliminated greater than $90 \%$ of PMNLs in the peripheral circulation as assessed by counts of Wright-stained smears of blood (Levine et al., 1984). Significant depletion of other leukocytes was also observed. 
Local accumulation of PMNLs in the skin of the paw was produced by intradermal injection of $10 \mu \mathrm{l}$ of $1 \%$ oyster glycogen (Baker Co., Phillipsburg, $\mathrm{NJ}$ ) in normal saline. The glycogen was injected $3 \frac{1}{2} \mathrm{hr}$ bcfore intradermal injection of test substances into the same site. This procedure has been shown histologically to produce a leukocytic infiltrate in rat skin that is-at $31 / 2 \mathrm{hr}$-almost completely accounted for by influx of PMNLs (Hurley, 1972; Wahba et al., 1984). The paw withdrawal threshold measured $31 / 2 \mathrm{hr}$ after glycogen injection was used as the base line threshold for calculating hyperalgesic effects in response to subsequent intradermal injections of test substances.

PMNLs for transfusion into hydroxyurea-treated rats and for in vitro PMNL stimulation experiments were obtained from peritoneal fluid of syngeneic rats $31 / 2 \mathrm{hr}$ after intraperitoneal injection of $1 \%$ glycogen $(15 \mathrm{ml})$. The glycogentreated rats were anesthetized with ether, and $15 \mathrm{ml}$ of ice-cold $\mathrm{Ca}^{2+}$. and $\mathrm{Mg}^{2+}$-free HBSS were injected into the peritoneal cavity. The rats then were sacrificed with an overdose of ether. Their abdomens were gently massaged, and the peritoneal fluid was removed through a midline incision in the linea alba and pooled for each group of rats. One milliiter of citrate buffer $(44 \mathrm{gm}$ of sodium citrate and $16 \mathrm{gm}$ of citric acid/liter) was added to each $15-\mathrm{ml}$ portion of pooled peritoneal fluid. PMNLs were recovered from the peritoneal fluid by centrifugation for $5 \mathrm{~min}$ at $600 \times \mathrm{g}$. The PMNLs were then washed twice and resuspended in HBSS. The purity of the PMNLs, as assessed by Wright-stained smears, was greater than $95 \%$.

For PMNL repletion experiments, approximately $10^{6}$ PMNLs were resuspended in $0.25 \mathrm{ml}$ of HBSS containing $0.3 \%$ rat albumin and injected into an indwelling carotid artery cannula $10 \mathrm{~min}$ before injecting test substances. To study the hyperalgesia-inducing factor produced by PMNLs, cell-free supernatant was prepared by incubating suspensions of $10^{7} \mathrm{PMNL} / \mathrm{ml}$ at $37^{\circ} \mathrm{C}$ for 15 min with $10^{-6} \mathrm{M} \mathrm{fMLP}$ (i.e., stimulated) and vehicle alone (i.e., unstimulated) for the control. After centrifugation of the suspensions, the supernatants were collected and further fractionated or stored at $-70^{\circ} \mathrm{C}$ for up to 4 days, until used for hyperalgesia testing.

To determine whether the hyperalgesia-inducing factor was also produced by human PMNLs, mixed leukocytes were obtained from citrate-anticoagulated blood of healthy human volunteers after dextran sedimentation of erythrocytes, as described (Goetzl and Austen, 1974). PMNLs of greater than $95 \%$ purity were isolated by centrifugation of the mixed leukocytes on Ficoll-Hypaque (Boyum, 1968). Purified human PMNLs were suspended at a concentration of approximately $10^{7}$ cells/ml in HBSS containing $0.1 \%$ oval bumin and $10 \mathrm{mM}$ HEPES (pH 7.4). Stimulated and control cell-free supernatants were prepared by the same procedure and suspended in the same vehicle used for rat PMNLS.

To obtain lipid and peptide-enriched fractions of the stimulated supernatants, two distinct methods of extraction were used. Peptides and other polar molecules were extracted from a $200-\mu \mathrm{l}$ aliquot of supernatant. The aliquot was acidified to $\mathrm{pH} 3$ with $0.4 \mathrm{~N} \mathrm{HCl}$ and then $800 \mu$ l of acetone were added. After refrigeration for $12 \mathrm{hr}$ at $4^{\circ} \mathrm{C}$, the aliquot was centrifuged at $100 \times g$ for $5 \mathrm{~min}$ at $40^{\circ} \mathrm{C}$, and the acetone supernatant was evaporated to dryness under nitrogen, followed by resuspension of the peptides in vehicle (Chang and Leeman, 1970). The supernatants from $10^{6}$ stimulated PMNLs were divided into four equal aliquots and injected into four rats.

The lipid fraction was obtained by acidifying a $900-\mu$ l aliquot of supernatant to $\mathrm{pH} 4$ with $0.4 \mathrm{~N} \mathrm{HCl}$, to which was added an equal volume of isopropanol, followed by extraction with 4 vol of ether. The ether layer was removed, evaporated to 5 to $10 \mu \mathrm{l}$, and resuspended in $\mathrm{MeOH}$ for storage at $-70^{\circ} \mathrm{C}$ under a nitrogen atmosphere (Matthay et al., 1984). Prior to use, the fraction was evaporated to dryness and resuspended in vehicle. Preliminary resolution of hyperalgesic lipid factors in supernatants from fMLP-stimulated and un stimulated PMNLs was performed by high pressure liquid chromatography (HPLC). The times of HPLC elution of synthetic prostaglandins, prostacyclins, thromboxanes, PAFacether, and the 8(R)all trans $\mathrm{LTB}_{4}$ product of 15-lipoxygenation of arachidonic acid were determined in the same solvent system to assess any relationship to the active lipid factors from stimulated PMNLS.

Time courses of the effect of different factors (see Fig. 1) were compared by a one-way analysis of variance of the tirne integral (i.e., area under the curve) for each treatment group. Mean values were compared statistically by a standard Student's $t$ test. The pretreatment of rats with indomethacin, hydroxyurea, or glycogen did not significantly effect paw withdrawal threshold ( $F=0.96, p=$ not significant by analysis of variance). For the paw withdrawal thresholds, taken immediately before injection of test substances (i.e., fMLP, $\mathrm{C}_{5 a}$. PMNL supernatants, or vehicle), the base line thresholds were: $4.96 \pm$ $0.45, n=96$ (mean \pm SE) for indomethacin; $5.14 \pm 0.63, n=80$ for hydroxyurea; $4.93 \pm 0.43, n=8$ for glycogen; and $5.03 \pm 0.62$ for untreated rats.

\section{Results}

Characteristics of hyperalgesia induced by chemotactic peptides. Both fMLP and $\mathrm{C}_{5 a}$, which are potent stimuli of chemotaxis and other PMNL functions, produced hyperalgesia with an identical time course when injected intradermally into the dorsum of the hindpaw of rats (Fig. 1). The hyperalgesia produced by intradermal injection of AMLP was detectable at a minimum dose of $0.044 \mathrm{ng}$ (Fig. 2), and at $0.44 \mathrm{ng}$ of $\mathrm{fMLP}$ or $0.4 \mathrm{ng}$ of $\mathrm{C}_{5 a}$ it produced hyperalgesia similar in magnitude and duration to that elicited by 20 ng of $L_{T}$ (Levine et al., 1984). The lowest effective dose of fMLP to elicit a detectable hyperalgesic effect is approximately one-tenth that found with $\mathrm{LTB}_{4}$ or $\mathrm{BK}$. The time course of hyperalgesia was, however, the same for all compounds. That is, fMLP, $C_{5 a}, L_{T B}$, and BK-induced hyperalgesia were all maximal by 20 min after intradermal injection.

Effect of inhibition of cyclo-oxygenase activity. Treatment of rats with the cyclo-oxygenase inhibitor indomethacin ( $2 \mathrm{mg} / \mathrm{kg}$, i.p.) or an equal volume of indomethacin vehicle, 30 min before intradermal injection of a peptide chemotactic factor, did not significantly modify the hyperalgesia elicited by $4.4 \mathrm{ng}$ of fMLP and $0.4 \mathrm{ng}$ of $\mathrm{C}_{5 \mathrm{a}}$ (Fig. $3)$. In contrast, the same dose of indomethacin completely blocks BK-induced hyperalgesia (Levine et al., 1984).

PMNL dependence of hyperalgesia. Hydroxyurea-induced depletion of circulating PMNLs, which had no effect acutely on either the health of the rats or on paw withdrawal threshold, prevented the hyperalgesia elicited by both fMLP (4.4 ng) and $\mathrm{C}_{5 a}(0.4 \mathrm{ng})$ (Fig. 4). The magnitude of the hyperalgesia produced in PMNL-depleted rats by intradermal injection of FMLP and $C_{5 a}$ did not differ significantly from that produced by intradermal injection of the same volume of vehicle in normal or PMNL-depleted rats. Intra-arterial administration of $10^{6}$ PMNLs to rats previously depleted of autologous circulating PMNLs, 10 min before intradermal injection of fMLP (0.44 ng), completely restored the hyperalgesic response (Fig. 4).

Local injection of glycogen, $3 \mathrm{hr}$ before intradermal injection of FMLP at the same site, markedly enhanced the hyperalgesia elicited

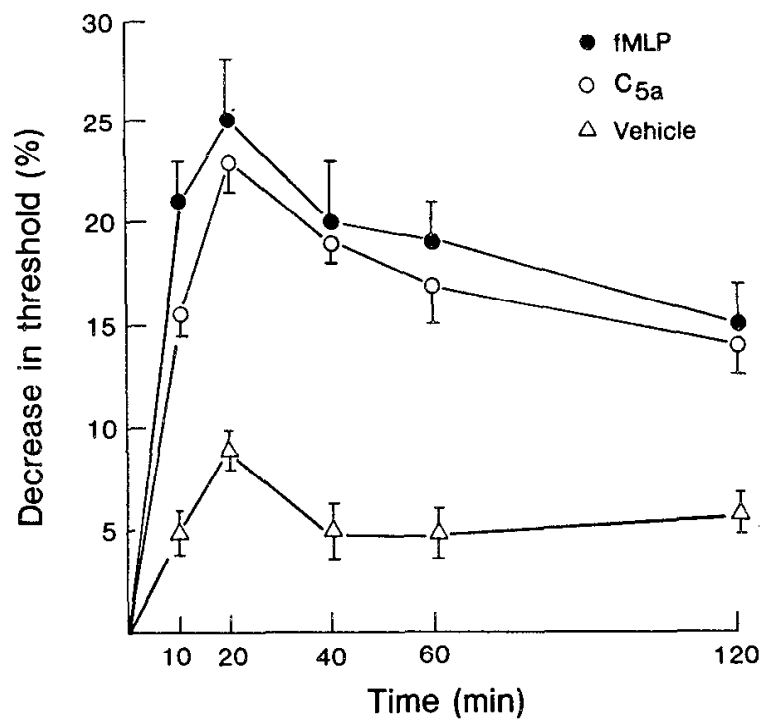

Figure 1. Time course of the effects of MLP (4.4 ng), $C_{5 a}(0.4 \mathrm{ng})$, and their vehicle on the nociceptive pressure thresholds in the rat paw. The fMLP, $\mathrm{C}_{5 \mathrm{a}}$, and vehicle were injected intradermally in the dorsum of the paw. Each error bar represents the mean \pm standard error of the results of studies of eight rats. A one-way analysis of variance of the time-weighted mean (i.e., integral over $120 \mathrm{~min}$ ) for these three groups (fMLP, $C_{5 a}$, and vehicle) was statistically significant $(F=8.85, p<0.01)$. Using a $t$ test to compare the area under the integral curves demonstrated that the hyperalgesia elicited by injections of $\mathrm{AMLP}$ and $\mathrm{C}_{5}$ were significantly greater than the hyperalgesia elicited by vehicle (both $p<0.01$ ). 


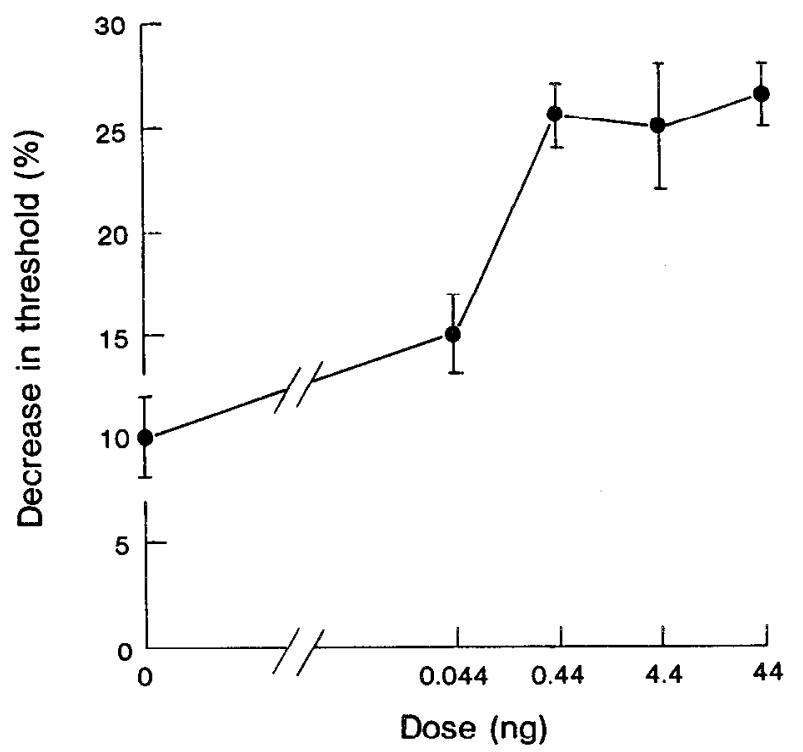

Figure 2. Concentration dependence of the effect of fMLP on nociceptive pressure thresholds. Measurements were made $20 \mathrm{~min}$ after the intradermal injection of $\mathrm{fMLP}$. Each point represents an average (mean $\pm \mathrm{SE}$ ) of eight experiments.

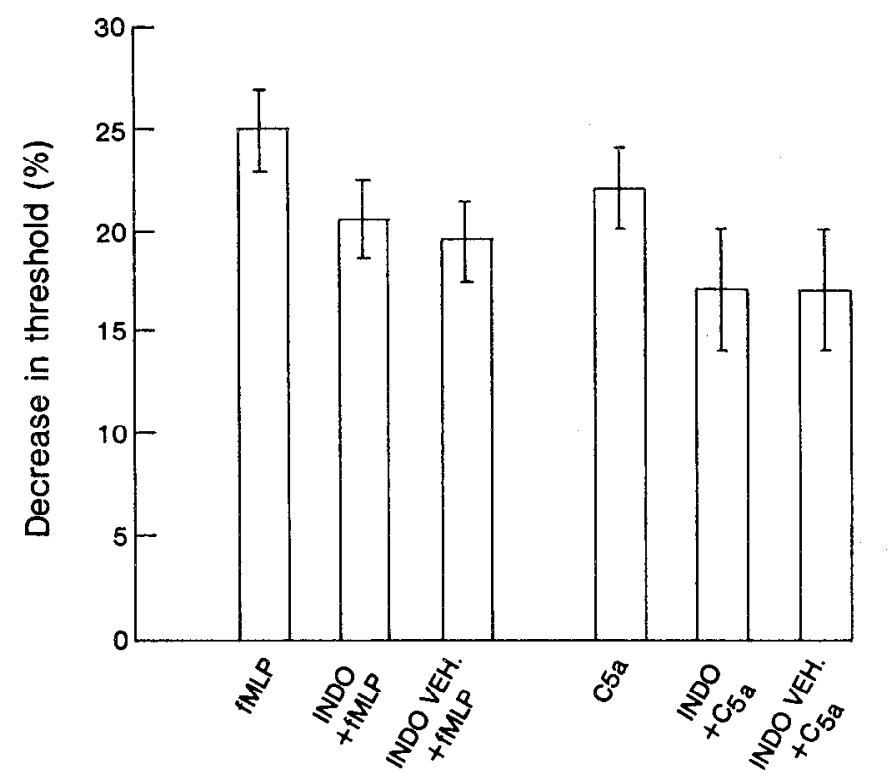

Figure 3. Lack of effect of indomethacin on the change in nociceptive pressure threshold induced by fMLP and $\mathrm{C}_{5 a}$. The fMLP. (4.4 ng) or $\mathrm{C}_{5 a}(0.4$ ng) was injecled intrademally 20 mir afler the administralion of indomeltracin (INDO) or vehicle (INDO VEH). Each bar depicts the average (mean $\pm \mathrm{SE}$ ) of eight experiments.

by the peptide chemotactic factors. Intradermal glycogen shifted the concentration dependence relationship for fMLP to the left by at least three orders of magnitude; that is, a maximal response could be elicited by the intradermal injection of $0.00044 \mathrm{ng}$ of fMLP. The magnitude of the peak hyperalgesic response $(38 \pm 4 \%$ ) at this lower dose $(0.00044 \mathrm{ng})$ was, in addition, significantly higher than the peak $(25 \pm 3 \%)$ produced by the higher amount $(4.4 \mathrm{ng})$ in rats that were not treated with glycogen $(p<0.01)$.

PMNL supernatant-induced hyperalgesia. The supernatant of $10^{7}$ fMLP-stimulated rat PMNLs produced significant hyperalgesia in PMNL-depleted rats $(\rho<0.05)$ (Fig. 5) that was similar in magnitude to that produced by $0.44 \mathrm{ng}$ of fMLP in normal rats (Fig. 2). In

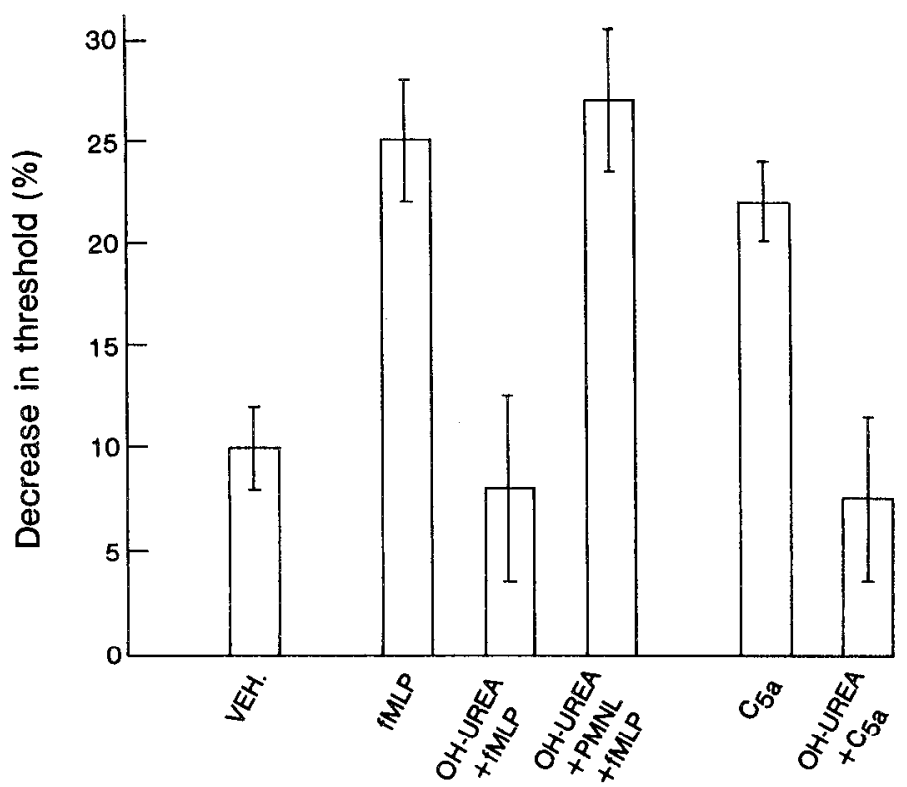

Figure 4. Effects of leukocyte depletion by hydroxyurea $(O H-U R E A)$ and leukocyte transfusion into PMNL-depleted rats (PMNL) on the responses of the nociceptive pressure threshold to fMLP and $C_{5 a}$. Measurements were made 20 min after intradermal injection of fMLP $(4.4 \mathrm{ng}), \mathrm{C}_{5 \mathrm{a}}(0.4 \mathrm{ng})$, or vehicle (VEH.) into a hindpaw. Each bar represents an average of eight experiments. Significant blunting of the hyperalgesic effect of $\mathrm{AMLP}(\rho<$ $0.05)$ and $C_{59}(p<0.025)$ on the pressure threshold was produced by PMNI depletion.

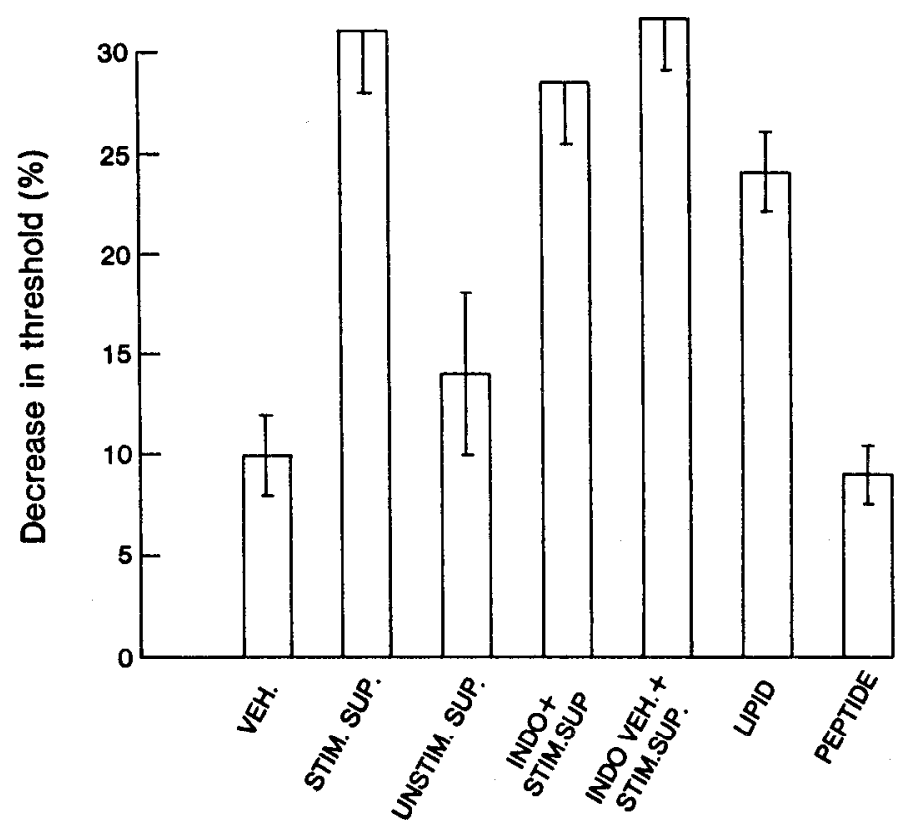

Figure 5. Effects of the supernatant of stimulated (STIM. SUP.) unstimulated (UNSTIM. SUP.) PMNLs, and of the lipid and peptide fraction of active supernatant from stimulated PMNLs, on the nociceptive threshold: and the lack of effect of indomethacin (INDO) or its vehicle (INDO VEH.) on supernatant-induced decrease in nociceptive threshold in PMNL-depleted rats. Measurements were made $20 \mathrm{~min}$ after intradermal injection of test substances. The supernatant of stimulated PMNLs and the lipid fraction of active supernatant produced significant hyperalgesia. Each bar represents an average (mean $\pm S E$ ) of eight experiments.

addition, the supernatant elicited some local soft tissue swelling. The supernatant of unstimulated rat PMNLs (Fig. 5) did not produce hyperalgesia. A significant level of hyperalgesia also was produced by the supernatant of $10^{7}$ fMLP-stimulated human PMNLs ( $30 \pm 4.5$; 
$n=8 ; p<0.025)$. Indomethacin did not significantly antagonize the hyperalgesia induced by supernatants of stimulated rat PMNLs (Fig. 5). Finally, when the stimulated PMNL supcrnatant was extracted by two separate techniques designed to yield peptides and lipids, significant $(\rho<0.01)$ biological activity was demonstrated only in the lipid fraction (Fig. 5). In preliminary experiments, the active factor in the lipid extract was further characterized by HPLC. The active HPLC fraction produced a peak hyperalgesic cffect in PMNL-depleted rats ( $22 \pm 2.1, n=8$ ) significantly greater than that produced by control supernatant from unstimulated PMNLs $(9 \pm 1.8, n=8 ; p$ $<0.01$ ). In addition, the HPLC fractions that coeluted with prostaglandins, prostacyclins, thromboxanes, and PAFacether did not produce significant hyperalgesia (maximum effect, $12 \pm 2.8, n=8$ ) with respect to supernatant from unstimulated PMNLs ( $\rho=$ not significant). The active factor coeluted with $8(\mathrm{R})$ all trans $\mathrm{LTB}_{4}$ but was not further characterized structurally.

\section{Discussion}

Two potent chemoattractants for and activators of PMNLs, $\mathrm{C}_{5 a}$ and $\mathrm{FMLP}$, not previously studied for their ability to affect sensory systems, both produced hyperalgesia, at low doses. The hyperalgesia produced was similar in time course to that recently reported for the hyperalgesia induced by a third PMNL-chemotactic factor, $\mathrm{LTB}_{4}$ (Levine et al., 1984). Since subnanogram doses of FMLP and $\mathrm{C}_{5 \mathrm{a}}$ induce a significant decrease in nociceptive thresholds, they are more potent, on a molar basis, than other well characterized hyperalgesic compounds including $\mathrm{BK}$ and $\mathrm{PGE}_{2}$ (Horton, 1963; Crunkhorn and Willis, 1971).

Involvement of PMNLs in the hyperalgesia elicited by $L_{T B}, \mathrm{fMLP}$, and $C_{5 a}$ is suggested by the ability of prior hydroxyurea treatment of rats, which depletes PMNLs (Wahba et al., 1984), to attenuate the hyperalgesia elicited by these compounds but not the hyperalgesia elicited by $\mathrm{BK}$ and $\mathrm{PGE}_{4}$ (Levine et al., 1984). Although hydroxyurea also depletes other circulating leukocytes and produces effects on non-hematopoietic cells (Calabresi and Parks, 1980), a specific contribution of PMNLs could be discerned from the transfusion studios in rats dopleted of endogenous PMNLs. Transfusion of PMNLs (>95\% pure) re-established the fMLP-induced hyperalgesia. Although it is possible that small numbers of non-PMNLS in the transfusions could account for the reconstitution of the hyperalgesic response, it is most likely due to reintroduction of PMNLs since the majority of "non PMNLs" obscrved on Wright stained smears of transfused cells were, in fact, cytoplasm without nuclei, and since fMLP, $\mathrm{C}_{5 a}$, and glycogen are potent PMNL-chemotactic and -activating factors that are preferentially active for PMNLs.

Although glycogen, a potent leukocyte chemoattractant, which in the time interval following injection employed in this study sclcctively attracts PMNLs, did not produce significant hyperalgesia, it produced a large shift in the dose dependency relationship for FMLP. induced hyperalgesia. When fMLP was administered into the same intradermal site $3 \frac{1}{2} \mathrm{hr}$ after glycogen, a significantly larger hyperalgesic effect was produced by one-thousandth of the dose previously required to elicit a maximal response. These findings provide independent confirmation of the contribution of PMNLs to the hyperalgesia elicited by fMLP and other PMNL chemoattractants. In addition, these findings suggest that the hyperalgesia elicited by chemotactic factors requires both attraction and activation of PMNLs.

The PMNL is the principal effector cell in the inflammatory reaction, accumulating at sites of inflammation to destroy antigenic material. Chemoattractants, such as $L_{T B}, f M L P$, and $C_{5 a}$ initiate a series of coordinated events in PMNLs including chemotaxis, secretion of lysozomal contents, and generation of superoxide anions (Snyderman and Pike, 1984). Although it is possible to elicit hyperalgesia in the absence of blood-borne elements (Lynn, 1977), the accumulation of PMNLs at sites of inflammation is commonly associated with marked hyperalgesia (e.g., gout) or pruritis (e.g. psoriasis), an allied pain sensation. Unfortunately, the regulation of PMNL function is complex and involves the release of a number of peptide and lipid factors which are potential candidates as mediators of hyperalgesia. There is little information, however, to suggest which of these factors are responsible for eliciting hyperalgesia, the characteristic sensory abnormality associated with inflammation.

The chemical nature of the substance(s) released from PMNLs, which elicit(s) hyperalgesia, was studied with supernatants trom PMNLs stimulated, in vitro, with fMLP. The supernatants of stimulated PMNLs produced hyperalgesia in PMNL-depleted rats. The hyperalgesic factor in these supernatants was found in its lipid fraction. Preliminary characterization of the active factor, by HPLC, has also demonstrated that it does not co-migrate on HPLC with prostaglandins, prostacyclins, thromboxanes, or PAFacether. That it does co-elute with $8(R)$ all trans $\mathrm{LTB}_{4}$ is important since it has recently been suggested that products of the 15-lipoxygenase pathway of arachidonic acid are formed by activated PMNLs, other leukocytes and epithelial cells (Jubiz et al., 1981; Maas et al., 1982; Hunter et al., 1985).

As previously reported for $\mathrm{LTB}_{4}$ (Levinc ct al., 1984), indomethacin failed to block $\mathrm{C}_{5 a}$ - and $\mathrm{fMLP}$-induced hyperaigesia. Thus, the mechanism by which these three chemotactic factors produce hyperalgesia is distinguished from the mechanism of the hyperalgesia elicited by BK and products of the cyclo-oxygenation of arachidonic acid such as E-type prostaglandins (Levine et al., 1984).

In conclusion, the PMNL-chemotactic factors $f M L P, C_{5 a}$, and $\mathrm{LTB}_{4}$ (Soter et al., 1983; Levine et al., 1984) all can produce hyperalgesia, the characteristic sensory abnormality associated with inflammation. Since one or more of these chemotactic factors are commonly present in inflammatory lesions, it is probable that this class of compounds could account for a large part of the hyperalgesia that develops in acute inflammation. The independence of the hyperalgesia elicited by these three substances from the cyclo-oxygenation of arachidonic acid and its dependence on PMNLs suggest that this type of hyperalgesia is mediated by a novel biochemical pathway. Preliminary characterization of the hyperalgesia-producing factor secreted by activated PMNLs; in response to these stimuli, suggests that it is a lipid. Pharmacological agents that block steps in this pathway may constitute a novel class of analgesic compounds.

Note added in proor. We have tested the effect of intradermal injection of the naturally occurring 15-lipoxygenase pathway product of PMNLs, $8(R)$ all trans $L T B_{4}$, and of $8(S)$ all trans $L T B_{4}$, a stereoisomer, on the pressure nociceptive threshold. The $8(R)$ all trans $L_{T B}$ isomer (100 ng) produced significant hyperalgesia when compared to the same volume of vehicle $(-20 \pm 2.3 \%, n=10$ versus $-5.1 \pm$ $2.3 \%, n=20, p<0.005$ by Student's $t$ test with Bonferroni's correction for multiple comparisons). In contrast, 8 (S)all trans $\mathrm{LTB}_{4}$ $(100 \mathrm{ng})$ produced significant hypoalgesia $(+9.0 \pm 3.5 \%, n=20$ versus $-5.1 \pm 2.3 \%, n=20, p<0.005$ ). These findings suggest that $8(\mathrm{R})$ all trans $\mathrm{LTB}_{4}$ could be a hyperalgesic substance released by PMNLs when they are stimulated with chemotactic factors. The findings that only the $8(\mathrm{R})$ all trans isomer produced hyperalgesia whereas the $8(S)$ isomer produced significant hypoalgesia suggests that this effect is receptor mediated and that the $8(\mathrm{~S})$ isomer may be an effective antagonist at this receptor site.

\section{References}

Boyum, A. (1968) Isolation of mononuclear cells and granulocytes from human blood. Scand. J. Clin. Lab. Invest. 21 (Suppl. 97): 77-89.

Calabresi, P. M., and R. R. Parks, Jr. (1980). Chemotherapy of neoplastic diseases. In The Pharmacological Basis of Therapeutics, A. G. Goodman, L. S. Goodman, and A. Gilman, eds., pp. 1256-1313, Macmillan, New York.

Chang, M. M., and S. E. Leeman (1970) Isolation of a sialogogic peptide from bovine hypothalamic tissue and its characterization as substance $P$ J. Biol. Chem. 245: 4784-4790.

Crunkhorn, P., and A. L. Willis (1971) Cutaneous reactions to intradermal prostaglandins. Br. J. Pharmacol. 41: 49-56.

Evans, W. O. (1964) A critical review of some new methods of animal analgesiometry. J. New Drugs 4: 179-187.

Ferreira, S. H. (1983) Prostaglandins: Peripheral and central analgesia. Adv. Pain Res. Ther. 5: 627-634. 
Ford-Hutchinson, A. W., M. A. Bray, M. V. Doig, M. E. Shipley, and M. J. H. Smith (1980) Leukotriene $B_{4}$, a potent chemokinetic and aggregating substance released from polymorphonuclear leukocytes. Nature 286: 264265.

Goetzl, E. J., and K. F. Austen (1974) Stimulation of human neutrophil leukocyte aerobic glucose metabolism by purified chemotactic factors. J. Clin. Invest. 53: 591-599.

Goetzl, E. J., and W. C. Pickett (1980) The human PMN leukocyte chemo tactic activity of complex hydroxy-eicosatetraenoic acids (HETEs). J. Immunol. 125: 1789-1791.

Horton, E. W. (1963) Action of prostaglandin $E_{1}$ on tissues which respond to bradykinin. Nature 200: 892-893.

Hunter, J. A., W. E. Finkbeiner, J. A. Nadel, E. J. Goetzl, and M. J. Holtzman (1985). Predominant generation of 15-lipoxygenase metabolites of arachidonic acid by epithelial cells from human trachea. Proc. Natl. Acad. Sci. U. S. A., in press.

Hurley, J. V. (1972) Acute Inflammation, pp. 109-116, Williams and Wilkins, Baltimore.

Johnson, A., and A. B. Malik (1980) Effect of granulocytopenia on extravascular lung water content after microembolization. Am. Rev. Respir. Dis. 122: $561-566$

Jubiz, W., O. Radmark, J. A. Lindgren, C. Malmsten, and B. Samuelsson (1981) Novcl leukotriencs: Products formed by initial oxygenation of arachidonic acid at $\mathrm{C}-15$. Biochem. Biophys. Res. Cummun. 99: 976-986.

Levine, J. D., W. Lau, G. Kwiat, and E. J. Goetzl (1984) Leukotriene $B_{4}$ produces hyperalyesia that is deperident on polyrnorphonuclear leukocytes. Science 225: 743-745.

Lynn, B. (1977) Cutaneous hyperalgesia. Br. Med. Bull. 33: 103-108.
Maas, R. L., A. R. Brash, and J. A. Dates (1982). Novel leukotrienes and lipoxygenase products from arachidonic acid. Adv. Prostaglandin Thromboxane Leukotriene Res. 9: 29-44.

Matthay, M. A., W. L. Eschenbacher, and E. J. Goetzl (1984) Elevated concentrations of leukotriene $B_{4}$ in pulmonary edema fluid of patients with adult respiratory distress syndrome. J. Clin. Immunol. 4: 479-483.

Randall, L. O., and J. J. Selitto (1957) A method for measurement of analgesic activity on inflammed tissue. Arch. Int. Pharmacodyn. 111: 409-419.

Shin, H. S., R. Snyderman, E. Friedman, A. Mellors, and M. D. Mayer (1968) Chemotactic and anaphylactic fragment, cleaved from the fifth component of guinea pig complement. Science 162: 361-363.

Showell, H. J., R. J. Freer, S. H. Zigmond, E. Schiffman, S. Aswanikumar, B. A. Corcoran, and E. L. Becker (1976) The structure-activity relations of synthetic peptides as chemotactic factors and inducers of lysosomal enzyme secretion for neutrophils. J. Exp. Med. 143: 1154-1169.

Snyderman, R., and M. C. Pike (1984) Transductional mechanisms of chemoattractant receptors on leukocytes. In Regulation of Leukocyte Function, R. Snyderman, ed., pp. 1-28, Plenum Press, New York.

Soter, N. A., R. A. Lewis, E. J. Corey, and K. F. Austen (1983) Local effects of synthetic leukotrienes $\left(\mathrm{LTC}_{4}, \mathrm{LTD}_{4}, \mathrm{LTE}_{4}\right.$, and $\left.\mathrm{LTB}_{4}\right)$ in human skin. $\mathrm{J}$ Invest. Dermatol. 80: 115-119.

Wahba, A. V., B. Barnes, and G. S. Lazarus (1984) Labeling of peripheral blood polymorphonuclcar lcukocytes with indium-111: A new method for the quantitation of in-vivo accumulation of PMNLs in rabbit skin. J. Invest. Dermatol. 82: 126-131.

Williarns, T. J. (1983) Interaclions belween prostaglandins, leukotrienes and other mediators of inflammation. Br. Med. Bull. 39: 239-242. 